\title{
Macroscopic Quantum System, Highly Correlated Electron State, and High-Temperature Superconductivity in Iron Pnictides
}

\author{
M. V. Krasinkova \\ Ioffe Physico-Technical Institute, Russian Academy of Sciences, St. Petersburg, Russia \\ Email: marinak@mail.ioffe.ru
}

Received 26 December 2013; revised 22 January 2014; accepted 14 February 2014

Copyright (C) 2014 by author and Scientific Research Publishing Inc. This work is licensed under the Creative Commons Attribution International License (CC BY). http://creativecommons.org/licenses/by/4.0/

(c) (i) Open Access

\section{Abstract}

The qualitative model of the high-temperature superconductivity suggested earlier for cuprates and based on the idea that the superconductivity is associated with delocalized $\pi$ bonding between ions is not only confirmed by experimental data on iron pnictides but is also improved. It is shown that the FeAs layer state is similar to that of a macroscopic quantum system characterized by a sandwich-type charge distribution in which negatively charged planes are two-dimensional electron crystals of pairs and positively charged planes are formed by positively charged ions. Superconductivity in such a system is accomplished by a two-dimensional Wigner crystal of bosons condensed into one and the same state. The crystal occupies a middle position with respect to charged planes in the sandwich structure, which leads to mutual compensation of all its interactions with all charged planes. The model can prove useful for development of the theory of superconductivity taking into consideration the highly correlated state of all valence electrons that manifests itself in formation of electron crystals with strong Coulomb interactions between them.

\section{Keywords}

Highly Correlated Electron State, Superconductivity, Coulomb Interactions, Electron Crystals, Boson Wigner Crystal, Macroscopic Quantum System

\section{Introduction}

Discovery of a new family of high-temperature superconductors based on iron pnictides and chalcogenides [1]-

How to cite this paper: Krasinkova, M.V. (2014) Macroscopic Quantum System, Highly Correlated Electron State, and High-Temperature Superconductivity in Iron Pnictides. Journal of Modern Physics, 5, 523-533. 
[4] attracted considerable attention of researchers. It was expected that comparison of properties of two families of superconducting materials (based on copper and iron) could get an insight into the mechanism of carrier pairing and reveal basic interactions responsible for superconductivity at rather high temperatures.

Research efforts have already led to some interesting conclusions. For instance, the highly contradictory data on the isotopic effect magnitude and sign in iron-based superconductors [5]-[7] have confirmed that electronphonon interactions are unlikely to be responsible for the carrier pairing mechanism. The absence of magnetic ordering in some iron-based superconducting materials [8] [9] and also a very low magnetic moment per $\mathrm{Fe}^{2+}$ in the range from 0.25 to $0.36 \mu \mathrm{B}$ [10] [11] which is almost an order of magnitude lower than the moment of 2.3 $\mu \mathrm{B}$ predicted theoretically [12] cast doubt on another explanation of the pairing mechanism suggested earlier for cuprates and including participation of short-range spin fluctuations [13].

However, the most intriguing finding of comparative analysis is that iron- and copper-based superconductors share a number of specific features, such as a layered structure, charge transfer, correlation between superconductivity and crystal lattice distortions, bell-shaped dependence of Tc on dopant concentration, dependence of Tc on pressure, a high second critical field $\mathrm{Hc}_{2}$, and the presence of anions with filled outer electron inert-gas shells and cations with unoccupied electron states.

The similarities between the two families raise the question as to whether the qualitative superconductivity model suggested earlier for cuprate superconductors [14] [15] is applicable to the new materials. The paper is devoted to finding an answer to this question.

\section{Specific Features of Chemical Bonding in Multicomponent Compounds Based on Iron Pnictides}

The idea underlying the high-Tc superconductivity model for cuprates [14] [15] is that characteristic properties of a material originate from specific features of chemical bonding because the character of bonding determines the state of valence electrons participating in it. Therefore, to define the electron state, the chemical bond character should be first of all considered. As will be shown below, such a consideration is able to give an insight into the reasons for and consequences of the highly correlated electron state in iron pnictides.

A small difference between electronegativities of iron and arsenic (less than 0.25) indicates that the bonding between these elements must be purely covalent. However, a charge transfer into the FeAs layer in the multicomponent compounds can change the character of bonding. Indeed, introduction of additional electrons into the FeAs layer converts an arsenic atom into a negatively charged $\mathrm{As}^{1-}$ ion, the outer electron shell of which is similar to that of an oxygen atom $\left(2 \mathrm{p}^{4}\right)$. Owing to this, $\mathrm{As}^{1-}$, like an oxygen atom, oxidizes iron to the $\mathrm{Fe}^{2+}$ state and is transformed into $\mathrm{As}^{3-}$ characterized by a fully occupied outer electron shell $\left(4 \mathrm{~s}^{2} 4 \mathrm{p}^{6}\right)$ and weakly bound outer electrons. The latter is evidenced by a very large ionic radius of $\mathrm{As}^{3-}(2.22 \AA)$, well above the atomic radius (1.39尺̊).

Thus, the negatively charged FeAs layer in the multicomponent compound proved to consist of $\mathrm{Fe}^{2+}$ and $\mathrm{As}^{3-}$ ions rather than of Fe and As atoms covalently bonded with each other. This fact is extremely important for understanding properties of the multicomponent compounds because 1 ) ionic compounds are characterized by ordering of local distortions, which promotes formation of quasi-one-dimensional (quasi-1D) structures (or ion chains) in the ionic quasi-two-dimensional (quasi-2D) lattice of the FeAs layer; 2) a strong electric field generated by $\mathrm{As}^{3-}$ around $\mathrm{Fe}^{2+}$ can cause a $\mathrm{Fe}^{2+}$ low-spin state that blocks participation of d-electrons in chemical bonding. (The low-spin state of $\mathrm{Fe}^{2+}$ was discussed for the FeP layers characterized by the same crystal structure as that of the FeAs layers [16] and for $\left.\mathrm{LaFeAsO}_{0.89} \mathrm{~F}_{0.11}[9]\right)$.

It is believed that small cations $\mathrm{Fe}^{2+}$ in the FeAs layer polarize large anions $\mathrm{As}^{3-}$, attract their weakly bound outer electrons and share them in the case of overlapping of ion electron states. The situation is similar to that in cuprates. The electron sharing by ions means that covalent components of mixed bonds are formed between them, and the electrons of the bonds prove to be localized by the polarization.

Let us see what covalent bonds can be formed between $\mathrm{Fe}^{2+}$ and $\mathrm{As}^{3-}$ in the FeAs layer consisting of $\mathrm{FeAs}_{4}$ tetrahedra.

$\mathrm{Fe}^{2+}$ with its outer electron $3 \mathrm{~d}^{6} 4 \mathrm{~s}^{0}$ shell can form four tetrahedrally oriented bonds (Figure 1(a)) only if it passes into the $\mathrm{sp}^{3}$ hybrid state that includes unoccupied $4 \mathrm{p}$ states. Such a hybridization keeps up a low-spin state with a symmetric $\mathrm{d}^{6}$ shell, and, hence, d-electrons do not participate in chemical bonding.

Concerning $\mathrm{As}^{3-}$ and its ability to form four bonds with four $\mathrm{Fe}^{2+}$ lying approximately in one plane (Figure 1(b)), its hybrid state must be either $d^{4} s$ or $d^{4} p$, where $d$ is the unoccupied $4 d$ states of $\mathrm{As}^{3-}$. This hybridization 


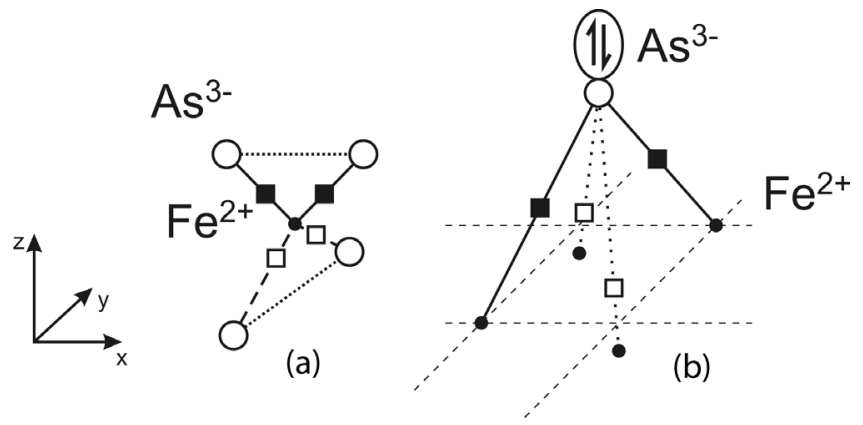

Figure 1. Coordination polyhedra of $\mathrm{Fe}^{2+}$ (a) and $\mathrm{As}^{3-}$ (b). Different lengths of $1 \mathrm{e}$ (dashed lines in (a) and dotted lines in (b)) and 2e (solid lines) bonds and one of two types of distortion of the $\mathrm{As}^{3-}$ pyramid base plane. Black squares show electron pairs, empty squares show unpaired electrons. The double arrow shows an unshared electron pair in the apical $\mathrm{As}^{3-}$ state.

gives rise to the $\mathrm{As}^{3-}$ coordination polyhedron in the form of a square pyramid typical of this ion in the compounds considered. According to Hund's rule, eight valence electrons of $\mathrm{As}^{3-}$ distributed among five hybrid states must form three singlet electron pairs that occupy three states and one triplet pair that occupies two remaining states. Therefore, $\mathrm{As}^{3-}$ can form two usual two-electron (2e) bonds (by using two singlet electron pairs) and two one-electron (1e) bonds (by using the triplet pair) with four $\mathrm{Fe}^{2+}$ ions. Thus, covalent bond components in the $\mathrm{As}^{3-}$ coordination polyhedron prove to be nonequivalent in energy and in lengths. (The presence of bonds of different lengths was experimentally confirmed by observation of two different Fe-As distances [11] [17]). A third singlet electron pair of $\mathrm{As}^{3-}$ must occupy the state at the square pyramid apex and remain an unshared pair, i.e., not participating in covalent bonding between ions (Figure 1(b)). Formation of nonequivalent bonds disturbs the spherically symmetric electron distribution in the $\mathrm{As}^{3-}$ shell and results in a highly nonuniform electron density distribution in its coordination polyhedron and a distortion of the polyhedron.

Since large $\mathrm{As}^{3-}$ ions cannot displace in the FeAs layer because of their dense packing, the coordination polyhedra with edges of different lengths can be formed only via $\mathrm{Fe}^{2+}$ displacements from their positions in the plane of the square pyramid base (Figure 1(b)), which is equivalent to the $\mathrm{Fe}^{2+}$ displacement from its tetrahedron center towards one of two unshared edges (Figure 2). This displacement contributes to formation of two short and two long bonds in each $\mathrm{FeAs}_{4}$ tetrahedron (or $\mathrm{AsFe}_{4}$ square pyramid) (Figure 1) and is accompanied by deviations of the angles between bonds from the ideal tetrahedron angle $\left(\beta=109.47^{\circ}\right)$ (Figure 2). The observation of the deviations [11] can be regarded as one of the proofs of $\mathrm{Fe}^{2+}$ displacements in their tetrahedra, though this experimental fact has not been associated so far with the idea of $\mathrm{Fe}^{2+}$ displacement.

The $\mathrm{Fe}^{2+}$ ordered displacements in neighboring tetrahedra in positive and negative directions of the $\mathrm{Z}$ axis (Figure 2) result in formation of two $\mathrm{Fe}^{2+}$ planes instead of one plane typically considered. Note that the FeAs layer itself is divided by the two $\mathrm{Fe}^{2+}$ planes into two parts (or two halves).

Two variants of $\mathrm{Fe}^{2+}$ ordered displacement in neighboring tetrahedra are possible (Figure 3). In the first case (Figure 3(a)) the interionic $\mathrm{Fe}^{2+}-\mathrm{Fe}^{2+}$ distance in the $\mathrm{As}^{3-}$ coordination pyramid base remains the same between all $\mathrm{Fe}^{2+}$, while in the second case (Figure 3(b)) there must be two different distances. The observation of two different $\mathrm{Fe}^{2+}-\mathrm{Fe}^{2+}$ distances for undoped LaOFeAs [11] and for the orthorhombic phase of $\mathrm{CaFe}_{2} \mathrm{As}_{2}$ [18] can be regarded as a direct confirmation of existence of two $\mathrm{Fe}^{2+}$ planes in these materials. The differences of 0.015 $\AA$ and $0.037 \AA$ in the $\mathrm{Fe}^{2+}-\mathrm{Fe}^{2+}$ distances obtained from the experimental data of [11] and [18], respectively, can be used to roughly estimate the ion displacement and the interplane distance. The estimation yields a displacement of $0.14 \AA$ and $0.22 \AA$ and a distance of $0.28 \AA$ and $0.44 \AA$ for $\mathrm{LaOFeAs}$ and $\mathrm{CaFe}_{2} \mathrm{As}_{2}$, respectively.

$\mathrm{The}_{\mathrm{Fe}} \mathrm{e}^{2+}$ ordered displacements are accompanied by ordering of nonequivalent bonds in the FeAs layer and formation of ion chains. In the case of the chess-board order of displacements, quasi-1D ion chains oriented in the direction of one of two diagonals in the square pyramid base are formed (Figure 4(b)). This bond ordering is typical of the tetragonal phase for which there must be no difference in the $\mathrm{Fe}^{2+}-\mathrm{Fe}^{2+}$ distances, consistent with experimental observations [18]. In the second case, ion chains bound by identical bonds become zig-zag-like not only along the $\mathrm{Z}$ axis, but also in the XY plane (Figure 4(a)) which is typical of the orthorhombic phase. 


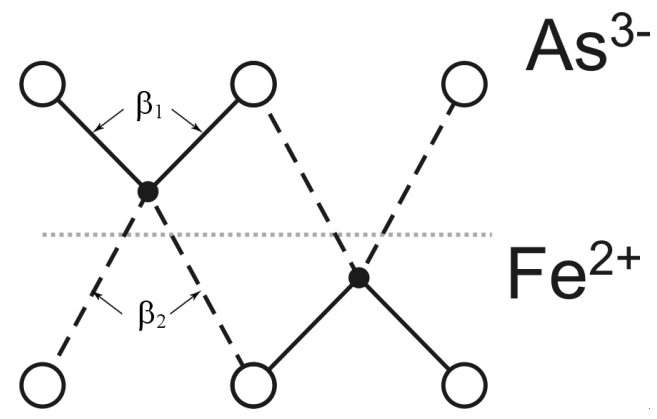

Figure 2. Schematic representation of $\mathrm{Fe}^{2+}$ displacement from the ideal tetrahedron centre (the dotted line shows the centre level) in two neighboring $\mathrm{FeAs}_{4}$ tetrahedra and formation of $1 \mathrm{e}$ and 2e bonds (dashed and solid lines, respectively).

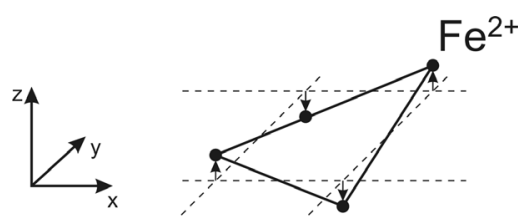

(a)

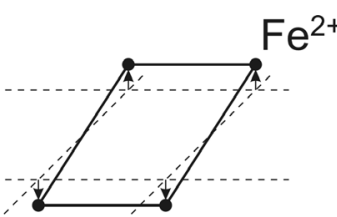

(b)

Figure 3. Two types of distortion of the $\mathrm{AsFe}_{4}$ square pyramid base resulting from $\mathrm{Fe}^{2+}$ ordered displacements in the tetragonal (a) and orthorhombic (b) phases.
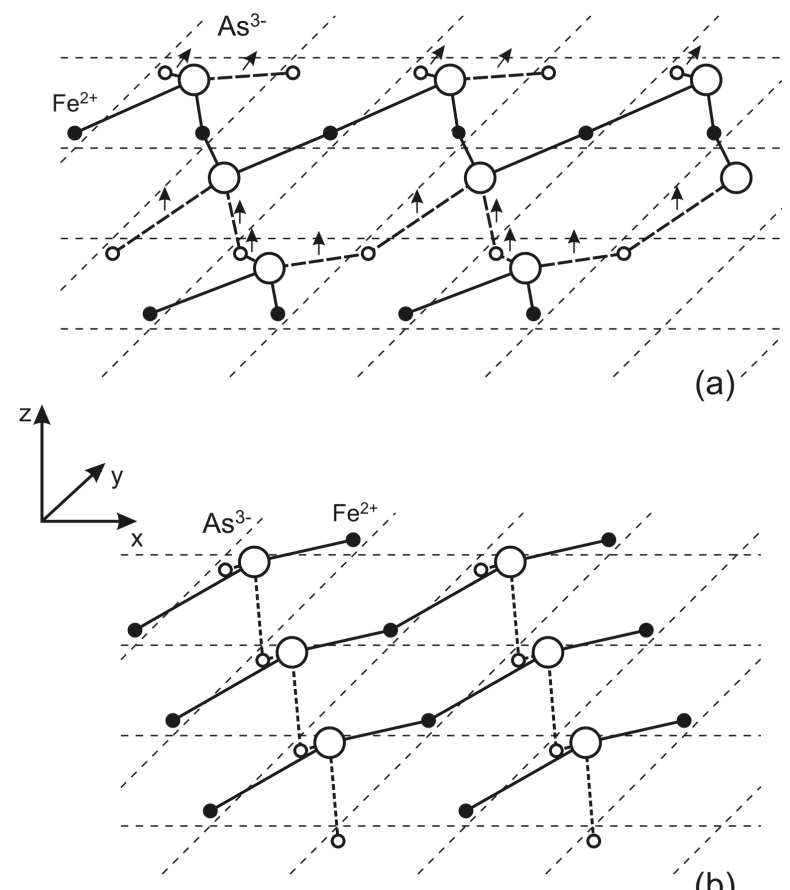

(b)

Figure 4. Ion chains bound by 2e bonds (solid lines) and separated from each other by static 1e bonds (dashed lines) in the orthorhombic phase (a) and by resonating 1e bonds (dotted lines) in the high-temperature tetragonal phase (b). The chains are shown for one half of the FeAs layer. Arrows show spins of unpaired electrons of $1 \mathrm{e}$ bonds which are ferromagnetically ordered in each chain. Small empty circles show $\mathrm{Fe}^{2+}$ belonging to the other FeAs layer half. 


\section{The State of Localized Valence Electrons Interacting with Each Other and a Sandwich-Type Charge Distribution in the FeAs Layer}

The nonequivalent bond formation results in a nonequivalence of the electron states. Three main states can be separated out: the state of singlet pairs; the state of unpaired electrons; and the state of unshared singlet pairs localized at anions. Note that there can also be another state of unshared pairs typical of the pairs formed from unpaired electrons in the low-temperature tetragonal phase (see Sections 4 and 5).

Let us consider in detail each of the electron states. The singlet pairs are formed in 2e bonds and are in the hybrid electron state resulting from overlapping of $\mathrm{As}^{3-}\left(\mathrm{d}^{4} \mathrm{~s}\right)$ and $\mathrm{Fe}^{2+}\left(\mathrm{sp}^{3}\right)$ hybrid states. The pairs are localized by cation-anion polarization in the space between ions and are distributed in the XY plane in a specific pattern that depends on both the 2e bond ordering in the ion coordination polyhedra and Coulomb interaction between these pairs at neighboring sites of their localization. As a result, spatial arrangements of electron pairs for tetragonal and orthorhombic phases are different. However, in general, the states of the pairs in both phases can be regarded as the states of $2 \mathrm{D}$ crystals of electron pairs located in each half of the FeAs layer. Owing to formation of $1 \mathrm{D}$ or quasi-1D chains of identical bonds, each of these two $2 \mathrm{D}$ crystals of electron pairs proves to be consisting of 1D (or quasi-1D) crystals each of which belongs to an individual ion chain (Figure 5(a) and Figure $5(c))$.

The valence electrons that form 1e bonds (because of this they are regarded as unpaired) are also in the hybrid electron states formed by overlapping of hybrid ion states. However, the degree of overlapping in this case is much lower (and the bond is longer) than that for 2e bonds considered above. This means that bonding of the unpaired electrons with ions is weaker. The unpaired electrons are also localized by cation-anion polarization, strongly interact with each other, and also form a 2D electron crystal in each half of the FeAs layer. Each of the

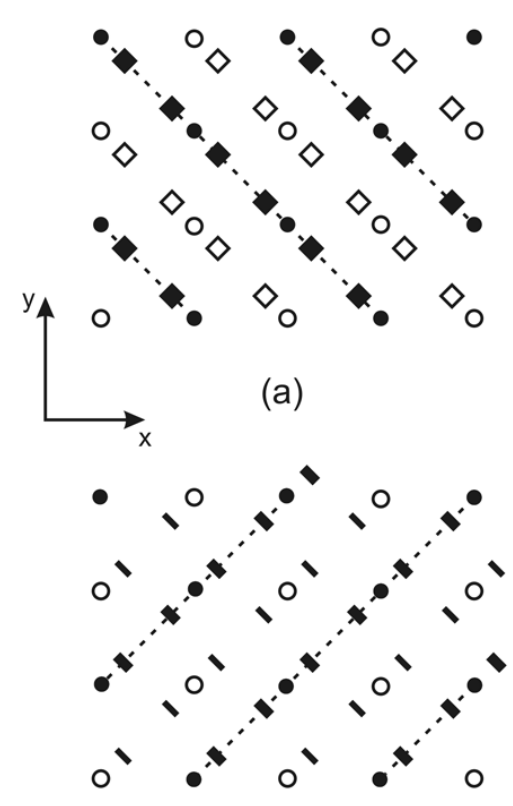

(b)

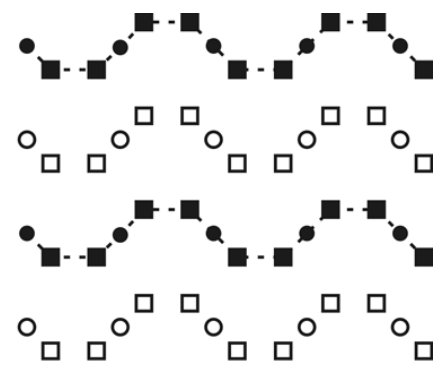

(c)

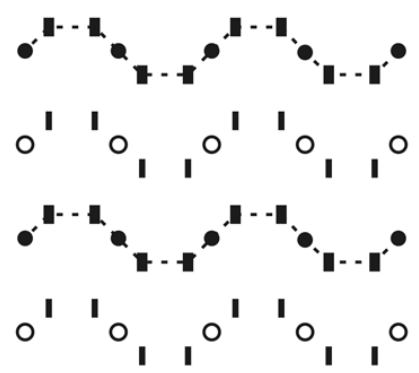

(d)

Figure 5. Projections (on the XY plane) of two 2D electron crystals of electron pairs forming 2e bonds (a) and two 2D electron crystals of unpaired electrons forming 1e bonds (b) in both parts of the FeAs layer together with the projections of two $\mathrm{Fe}^{2+}$ planes for the tetragonal phase. The thick dashes and black squares and circles show unpaired electrons, electron pairs, and $\mathrm{Fe}^{2+}$ ions, respectively, which are in one part of the layer. The thin dashes, empty squares and circles show the same for the other part of the layer. Similar schematic pictures are given for the orthorhombic phase ((c), (d)). The dotted lines are aid for the eye. Distances between 1D and quasi-1D electron crystals in each part of the FeAs layer are $3.9 \AA$ for (a, b) and $5.6 \AA$ for (c, d). $\mathrm{As}^{5+}$ are not shown to make the figure simpler. 
2D crystals is divided into 1D or quasi-1D crystals (due to the existence of ion chains) (Figure 5(b) and Figure 5(d)). The spatial patterns of the 2D crystals of unpaired electrons and pairs are similar to each other in both phases, and the crystals are displaced in the FeAs layer with respect to each other both along the $\mathrm{Z}$ axis and in the XY plane. Note that the 2D crystal state is preserved for unpaired electrons even after transition of the 1e bonds into a resonance state (for instance, in the high-temperature tetragonal phase) [19].

There is one more specific feature of the unpaired electrons that should be pointed out. We regard them as unpaired only because they form 1e bonds. Actually, every two unpaired electrons form a triplet pair if they occupy two identical electron states in one $\mathrm{As}^{3-}$ coordination polyhedron. Therefore, a crystal of unpaired electrons can also be referred to as a crystal of triplet pairs. The first presentation is convenient for understanding the general picture of electron-electron interactions in the FeAs layer, the second one is useful for understanding physical properties of materials (metal-like conductivity and magnetic ordering).

Note that there is a strong Coulomb interaction between the 2D electron crystals (of unpaired electrons and pairs), which is a consequence of Coulomb interaction between the unpaired electrons and pairs in each ion coordination polyhedron (Figure 1).

The third group of valence electrons includes unshared singlet electron pairs localized in apical hybrid $\mathrm{As}^{3-}$ states. Owing to Coulomb repulsion between pairs at neighboring $\mathrm{As}^{3-}$, these pairs form 2D crystals of unshared electron pairs that create negatively charged surfaces of the FeAs layer. This provides purely ionic bonds between the layer and neighboring positively charged layers with another chemical composition.

Analysis of the valence electron state shows that, though electrons are divided into three groups in accordance with the character of the bonds they form, they have much in common, i.e., 1) all of them are characterized by localization and bonding with ions which can be strong (electron pairs or unshared pairs) and weak (unpaired electrons and unshared pairs formed by them at doping and under pressure), and 2) all of them form 2D electron crystals with quantized energy levels. Projections of electron crystals and $\mathrm{Fe}^{2+}$ planes on the XY plane are shown in Figure 5. The Coulomb interactions between all electron crystals formed by different groups of electrons result in ordering of all charged planes in the FeAs layer (including positively charged ion planes). The ordering leads to formation of a sandwich-type structure of charge distribution. The sandwich-type structure consisting of 10 charged planes which includes 6 planes of 2D electron crystals and 4 planes of positively charged ions is presented in Figure 6(a). (Here the arsenic ion is regarded as $\mathrm{As}^{5+}$ rather than $\mathrm{As}^{3-}$ because we

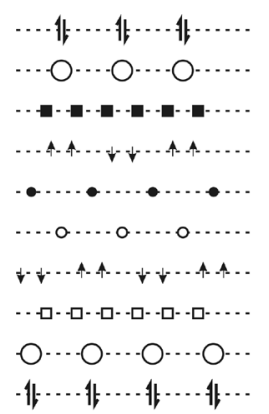

(a)

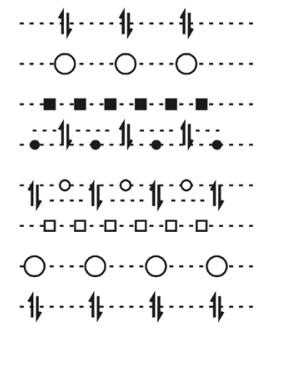

(b)

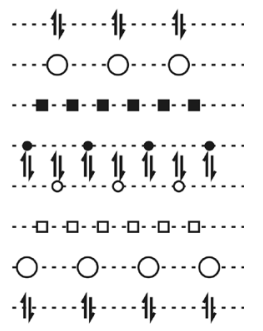

(c)

Figure 6. A sandwich-type charge distribution along the $\mathrm{Z}$ axis in the FeAs layer and its change with increasing Coulomb interaction between electron crystals. The sequence of the planes in (a) is as follows (from top to bottom): $2 \mathrm{D}$ crystal of unshared pairs, $\mathrm{As}^{5+}$ plane, 2D crystal of pairs of 2e bonds, 2D crystal of unpaired electrons (or triplet pairs), $\mathrm{Fe}^{2+}$ plane. The order of planes is reverse in the layer second part. The designations are the same as in Figure 5. Large empty circles show $\mathrm{As}^{5+}$. Figure 6(a) shows charge distribution in the FeAs layer in the orthorhombic phase (no doping and pressure application) characterized by two 2D crystals of unpaired electrons. Figure 6(b) shows an intermediate, probably unstable state characterized by two 2D Wigner crystals of unshared pairs (or bosons) shown by double arrows near the $\mathrm{Fe}^{2+}$ planes. Figure 6(c) shows the superconducting state characterized by the united 2D Wigner crystal of bosons situated between two $\mathrm{Fe}^{2+}$ planes. 
consider its valence electrons separately as forming their own planes, i.e., planes of 2D electron crystals). The sandwich structure is characterized by very small inter-plane distances along the $\mathrm{Z}$ axis (of the order of tenths of $\AA$ ), which is an order of magnitude lower than the minimal inter-charge distances in the planes (it is about $3.9 \AA$ in the $\mathrm{Fe}^{2+}$ and planes, $1.65 \AA$ in the crystal of unpaired electrons, and $1.96 \AA$ in the crystal of electron pairs). This confirms the existence of a strong Coulomb interaction between all charged planes along the $\mathrm{Z}$ axis. Moreover, electron crystals of one half of the layer can experience the Coulomb interaction with the crystals of the second half due to large inter-charge distances in the planes and relative displacements of charges (in the XY plane) in both parts of the FeAs layer.

\section{Singlet Pairing of Unpaired Electrons and Formation of Wigner Crystals of Bosons under Doping}

It is known that a partial substitution (10 percent) of oxygen by fluorine in LaOFeAs accompaned by introduction of additional electrons into the FeAs layer gives rise to superconductivity. It can be supposed that each introduced electron is paired with one of two unpaired electrons of $\mathrm{As}^{3-}$ participating in formation of 1e bonds. The pair formation breaks the 1e bond, bonding between the ions becomes purely ionic, and $\mathrm{Fe}^{2+}$ gets an unoccupied electron state. The singlet pair localizes at $\mathrm{As}^{3-}$ and becomes a new unshared pair of $\mathrm{As}^{3-}$ (in addition to the unshared one in its apical electron state). The bonding of the new pair with $\mathrm{As}^{3-}$ becomes much weaker than that of its parent unpaired electron due to enhanced Coulomb repulsion from the 2e bond pairs present in the $\mathrm{As}^{3-}$ coordination polyhedron (Figure 1(b) and Figure 7). It is quite possible that the new pair will even pass from the $\mathrm{d}^{4} \mathrm{~s}$ state into a higher-energy $\mathrm{As}^{3-}$ state formed by adding a part of the $\mathrm{p}$ state (or even into a new $\mathrm{d}^{4} \mathrm{p}$ state).

Moreover, the formation of a singlet pair with a higher energy in the crystal of unpaired electrons will be accompanied by increasing Coulomb repulsion not only between this pair and the neighboring unpaired electrons but also between the unpaired electron crystal and the neighboring electron crystals of pairs (Figure 6(a) and Figure 7). The increased Coulomb repulsion results in a local perturbation in the balance of Coulomb interactions between electron crystals in the sandwich charge distribution. It can be supposed that when the concentration of such perturbations becomes critical (or, in other words, the Coulomb interaction between electron crystals reaches a critical value), a new charge distribution in the sandwich structure arises and results in a collective change in the state of all unpaired electrons, i.e., singlet pairs are formed by all unpaired electrons (throughout the 2D unpaired electron crystal). This means that the 2D unpaired electron crystal is replaced by the 2D crystal of unshared pairs which is at a higher energy level. Since the unpaired electrons also form triplet pairs, such a replacement can be considered as a transition from triplet pairing to the singlet one.

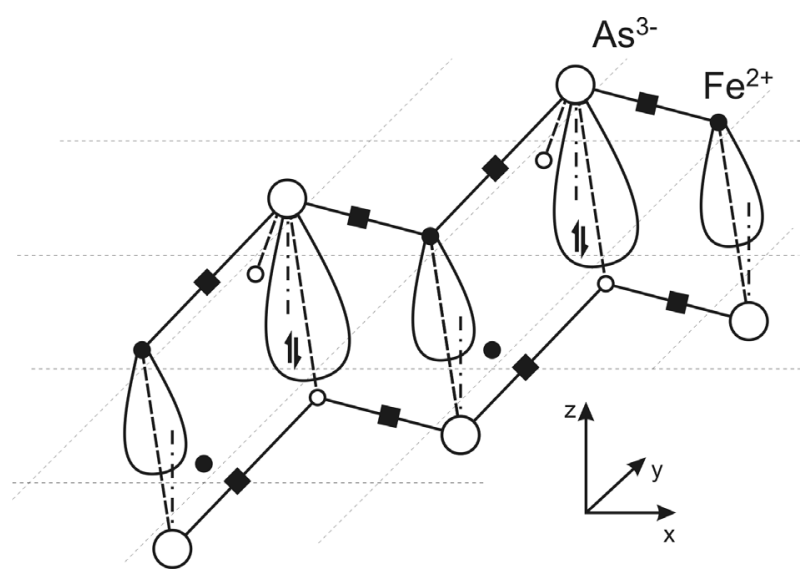

Figure 7. Mutual arrangement of electron states of the new unshared pairs and $\mathrm{Fe}^{2+}$ unoccupied states and their alternation along the chain of covalently bonded ions, and also the position of the unshared pair (double arrow) with respect to the electron pairs in the coordination polyhedron of its $\mathrm{As}^{3-}$ and two pairs of nearest neighboring $\mathrm{As}^{3^{-}}$situated in the second part of the FeAs layer (All pairs are shown by black squares). 
Since the unshared pairs are real particles, have zero spins and, as shown below, are able to condense into one and the same state (i.e., they obey the Bose-Einstein statistics), they can be regarded as zero-spin bosons and their 2D crystal can be regarded as a 2D Wigner crystal of bosons. Here the notion of Wigner crystal introduced earlier by E. P. Wigner for electrons is extended to cover bosons because the classical Wigner crystal and the crystal of bosons exhibit similar behaviors. For instance, they can be delocalized as a whole, and while the Wigner crystal of electrons gives rise to the Frölich conductivity, the Wigner crystal of bosons, as will be shown below, leads to the superconductivity.

\section{Singlet Pairing of Unpaired Electrons and Formation of Wigner Crystals of Bosons under Pressure}

The orientation of weaker (and much more compressible) 1e bonds in the FeAs layer must lead to a highly anisotropic layer shrinkage under hydrostatic pressure. The shrinkage can occur via $\mathrm{As}^{3-}$ displacements along the $\mathrm{Z}$ axis towards the $\mathrm{Fe}^{2+}$ planes in both parts of the layer (Figure 8). Exactly this shrinkage anisotropy was observed experimentally [18]. In addition, as can be seen from Figure 8, the shrinkage must be accompanied by a decrease in the angle $\beta$ between 1e (or ionic bonds substituting them) and 2e bonds in the $\mathrm{Fe}^{2+}$ coordination polyhedron, which was also observed experimentally [18].

A decrease in angle $\beta$ results in an increase of Coulomb repulsion between unpaired electrons and electron pairs of 2e bonds in the $\mathrm{Fe}^{2+}$ polyhedra (Figure 8) or, in general, between their electron crystals. This increases the energy of unpaired electrons and, probably, can lead to their transition from the $\mathrm{d}^{4} \mathrm{~s}$ state of $\mathrm{As}^{3-}$ into, for instance, a higher-energy $\mathrm{d}^{4} \mathrm{p}$ state and from the triplet pairing to a singlet one. As a result, a singlet electron pair appears in one of two $\mathrm{As}^{3-}$ states occupied earlier by two unpaired electrons, and this pair becomes a new unshared pair of $\mathrm{As}^{3-}$ (or boson), just like at doping. The boson formation is accompanied by transformation of 2D unpaired electron crystals into 2D Wigner crystals of bosons (Figure 6(b)) in both halves of the FeAs layer.

\section{Boson Condensation and a Unique State of the FeAs Layer}

As shown above, enhancement of Coulomb interaction in the FeAs layer (at doping or under pressure) results in formation of bosons and two 2D Wigner crystals of bosons (Figure 6(b) and Figure 9(a)). These crystals are plane-square lattices with the square side equal to the distance (d) between the nearest $\mathrm{As}^{3-}$ lying in one and the same plane $(3.9 \AA)$ and are displaced by $\mathrm{d} / 2$ with respect to each other along the $\mathrm{X}$ and $\mathrm{Y}$ axes in different halves of the layer. Note that each of these two boson crystals consists of 1D Wigner crystals because bosons belong to $\mathrm{As}^{3-}$ ions of separate chains of covalently bonded ions $\left(\mathrm{As}^{3-}\right.$ and $\mathrm{Fe}^{2+}$ ) (Figure 7).

Boson formation is accompanied by the emergence of unoccupied hybrid $\mathrm{Fe}^{2+}$ states which are partly in the space between the $\mathrm{Fe}^{2+}$ planes (Figure 8). These states are oriented in the same direction as the states of bosons, i.e., along one of lateral edges of the $\mathrm{As}^{3-}$ coordination pyramid. Moreover, the unoccupied $\mathrm{Fe}^{2+}$ states and the states of bosons alternate along the entire ion chain length (Figure 7).

As temperature decreases and the crystal lattice of the FeAs layer shrinks, the Coulomb interaction between the Wigner boson crystals and the neighboring electron crystals of pairs increasies and forces the 2D Wigner

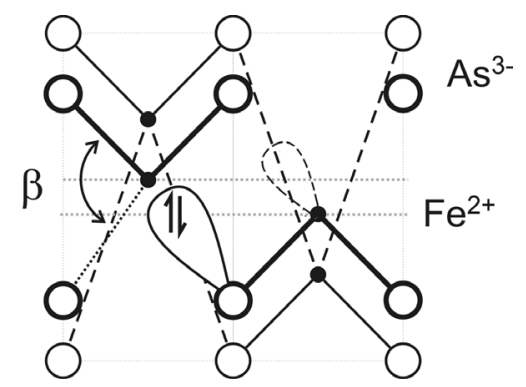

Figure 8. Deformation of two neighboring $\mathrm{Fe}^{2+}$ tetrahedra under pressure $\left(\mathrm{As}^{3-}\right.$ displacement, a decrease in angle $\beta$, and formation of an unshared pair (boson) in the $\mathrm{As}^{3-}$ state and $\mathrm{Fe}^{2+}$ unoccupied state) resulting from substitution of $1 \mathrm{e}$ bonds by ionic ones. $\mathrm{As}^{3-}$ displaced along the $\mathrm{Z}$ axis are shown by heavy circles. 


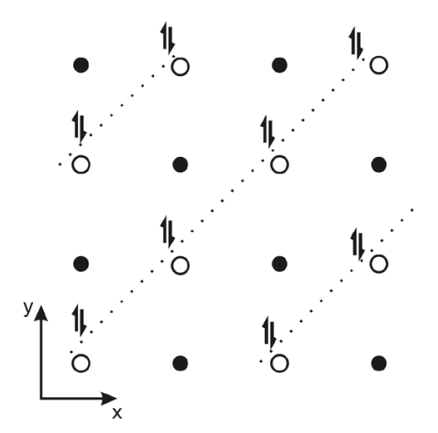

(a)

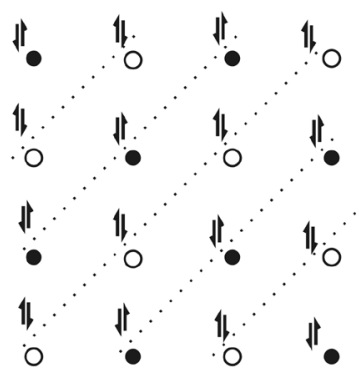

(b)

Figure 9. Projections (on the XY plane) of 2D Wigner crystal of bosons (prior to the united crystal formation) in one half of the FeAs layer (a) and of the united 2D Wigner crystal of bosons (b) together with projections of two planes of $\mathrm{Fe}^{2+}$ ions shown by black and empty circles. The dotted lines show the orientation of the 1D Wigner crystals constituting these 2D Wigner crystals. Different orientations of the double arrows designating bosons in (b) show that they belong to different ion chains.

boson crystals to move from the $\mathrm{As}^{3-}$ planes towards the $\mathrm{Fe}^{2+}$ planes (in both halves of the FeAs layer) (Figure 6(b)). In all probability, when these crystals fall into the space between the $\mathrm{Fe}^{2+}$ planes, lateral or $\pi$ overlapping of unoccupied $\mathrm{Fe}^{2+}$ states and $\mathrm{As}^{3-}$ states occupied by bosons can occur. This results in formation of delocalized $\pi$ orbitals extending along all chains of covalently bonded ions. Such an orbital is nothing else but a quasi-1D electron extending along the chain. As a result, all bosons of each 1D Wigner crystal turn out to be in exactly the same state. In other words, the delocalized $\pi$ orbital formation is accompanied by boson condensation in each 1D Wigner crystal of bosons. Hybridization of ion states at their overlapping leads to a boson energy increase which is likely to compensate for the bonding energy of bosons with individual $\mathrm{As}^{3-}$. This means that the bosons constituting the 1D Wigner crystal are shared by $\left(\mathrm{As}^{3-}\right.$ and $\mathrm{Fe}^{2+}$ ) ions in the chain. It would seem, the 1D Wigner boson crystal can be delocalized as a whole along the chain. However, the Coulomb interactions between the 1D Wigner boson crystals in the 2D Wigner crystal (i.e., in the XY plane) (Figure 9(a)) and also with the neighboring 2D crystals of pairs (i.e., along the $\mathrm{Z}$ axis) (Figure 6(b) and Figure 7) make the delocalization impossible.

It is quite possible that, together with the ion state overlapping in the space between the $\mathrm{Fe}^{2+}$ planes, a coalescence of two 2D Wigner crystals of bosons (from both halves of the FeAs layer) and formation of a united crystal of bosons occur (Figure 6(c)). This is accompanied by increasing Coulomb interaction between the 1D boson Wigner crystals in the united 2D crystal (Figure 9). In addition, in the space between the $\mathrm{Fe}^{2+}$ planes the united 2D Wigner crystal of bosons proves to be in a middle position (relative to the charged planes in the sandwich structure) (Figure 6(c)) in which its Coulomb interactions with all positively and negatively charged planes surrounding it compensate each other. This means there is nothing to inhibit delocalization of the united 2D Wigner boson crystal as a whole (along all $\pi$ orbitals, i.e., without any resistance) and, hence, the delocalization can provide superconductivity in the FeAs layer.

Concerning the other fundamental characteristic of a superconductor, i.e., the magnetic field expulsion from the interior during its transition to the superconducting state (the Meissner effect), the model of superconductivity based on $\pi$ bonding is able to explain the origin of persistent currents. Though bosons in the FeAs layer form the 2D Wigner crystal their state cannot be considered as a state of rest. On the contrary, bosons are in a constant rotation around their positions in the plane of their 2D Wignar crystal (or, in other words, around their mass centers). The rotation is induced by the Coulomb interaction between neighboring bosons because they have a dumb-bell shape (two electrons constituting the boson are separated by a distance). The rotation is in synchronism in the boson crystal plane state and is characterized by the same angular velocity for all bosons but opposite directions of the velocity and the $\pi / 2$ phase shift for neighboring bosons. From the chemical point of view the boson rotation provides the $\pi$ bonding between $\mathrm{As}^{3-}$ and two neighboring $\mathrm{Fe}^{2+}$ ions. The boson rotation induces persistent currents which are indispensable to the Meissner effect. This problem will be considered in detail elsewhere. 
Thus, the FeAs layer state is characterized by 1) a well-defined sandwich-type charge distribution; 2) strong Coulomb interactions between all charged planes of the sandwich structure; 3) quantized electron crystal energy levels; 4) a possibility of delocalization of 2D Wigner crystal of bosons without any resistance; and 5) the presence of persistent currents reminding, in a sense, electron currents in atoms. All these features point to a unique state of the FeAs layer and make it similar to a macroscopic quantum system.

\section{Conclusions}

Thus, the qualitative (not quantitative at the moment) model of high-temperature superconductivity suggested earlier for cuprates has been further developed owing to the analysis of experimental data for iron pnictides. It has confirmed that Coulomb interaction between ions resulting in sharing of electrons and electron pairs between ions leads to electron localization and that strong Coulomb interactions between the localized electrons and between electron pairs and also with each other result in a highly correlated state of all valence electrons. The state manifests itself in formation of electron crystals of unpaired electrons and pairs which build up a sandwich-type charge distribution in the FeAS layer. The layer becomes similar to a macroscopic quantum system.

Superconductivity in such a system emerges when doping or pressure and/or temperature lowering leads to an increase in the Coulomb interaction between electron crystals and thus gives rise to transformation of 2D unpaired electron crystals into the 2D Wigner crystal of bosons condensated into one and the same boson state.

We hope that the qualitative model of superconductivity can prove useful for the search for new superconducting materials and development of the theory of superconductivity taking into account the highly correlated state of valence electrons and also strong Coulomb interactions between electron crystals. A new mathematical approach differing from that used for fermionic systems can be needed to get quantitative estimates of interactions in such a macroscopic quantum system.

\section{Acknowledgements}

The author would like to acknowledge very useful discussions of characteristic properties of superconductivity in materials with the strongly correlated electron state with Academician Yu.V. Kopaev and Prof. K.D. Tsendin.

\section{References}

[1] Kamihara, Y., Watanabe, T., Hirano, M. and Hosono, H. (2008) Journal of the American Chemical Society, 130, 32963297. http://dx.doi.org/10.1021/ja800073m

[2] Ren, Z.A., Lu, W., Yang, J., Yi, W., Shen, X.L., Li, Z.C., Che, G.C., Dong, X.L., Sun, L.L., Zhou, F. and Zhao, Z.X. (2008) Chinese Physics Letters, 25, 2215-2216. http://dx.doi.org/10.1088/0256-307X/25/6/080

[3] Hsu, F.C., Luo, J.Y., Yeh, K.W., Chen, T.K., Huang, T.W., Wu, P.M., Lee, Y.C., Huang, Y.L., Chu, Y.Y., Yan, D.C. and Wu, M.K. (2008) Proceedings of the National Academy of Sciences, 105, 14262-14264. http://dx.doi.org/10.1073/pnas.0807325105

[4] Mizuguchi, Y., Tomioka, F., Tsuda, S., Yamaguchi, T. and Takano, Y. (2008) Applied Physics Letters, 93, 152505. http://dx.doi.org/10.1063/1.3000616

[5] Shirage, P.M., Kihou, K., Miyazawa, K., Lee, C.H., Kito, H., Eisaki, H., Yanagisawa, T., Tanaka, Y. and Iyo, A. (2009) Physical Review Letters, 103, 257003. http://dx.doi.org/10.1103/PhysRevLett.103.257003

[6] Liu, R.H., Wu, T., Wu, G., Chen, H., Wang, X.F., Xie, Y.L., Ying, J.J., Yan, Y.J., Li, Q.J., Shi, B.C., Chu, W.S., Wu, Z.Y. and Chen, X.H. (2009) Nature, 459, 64-67. http://dx.doi.org/10.1038/nature07981

[7] Shirage, P.M., Miyazawa, K., Kihou, K., Kito, H., Yoshida, Y., Tanaka, Y., Eisaki, H. and Iyo, A. (2010) Physical Review Letters, 105, 037004. http://dx.doi.org/10.1103/PhysRevLett.105.037004

[8] Blachowski, A., Ruebenbauer, K., Zukrowski, J., Przewoznik, J. and Marzec, J. (2010) Journal of Alloys and Compounds, 505, L35-L37. http://dx.doi.org/10.1016/j.jallcom.2010.06.118

[9] Kitao, S., Kobayashi, Y., Higashitaniguchi, S., Saito, M., Kamihara, Y., Hirano, M., Mitsui, T., Hosono, H. and Seto, M. (2008) Journal of the Physical Society of Japan, 77, 103706. http://dx.doi.org/10.1143/JPSJ.77.103706

[10] Chen, Y., Lynn, J.W., Li, J., Li, G., Chen, G.F., Luo, J.L., Wang, N.L., Dai, P., de la Gruz, C. and Mook, H.A. (2008) Physical Review B, 78, 064515. http://dx.doi.org/10.1103/PhysRevB.78.064515

[11] de la Cruz, C., Huang, Q., Lynn, J.W., Li, J., Ratcliff, W., Zarestky, J.L., Mook, H.A., Chen, G.F., Luo, J.L., Wang, N.L. and Dai, P. (2008) Nature, 453, 899-902. http://dx.doi.org/10.1038/nature07057

[12] Cao, C., Hirschfeld, P.J., Cheng, H.P. (2008) Physical Review B, 77, 220506. 
http://dx.doi.org/10.1103/PhysRevB.77.220506

[13] Manske, D. (2004) Theory of Unconventional Superconductors. In: Hohler, G., Ed., Springer Tracts in Modern Physics, Vol. 202, Springer Verlag, Berlin, 1-228.

[14] Krasinkova, M.V. (2006) Physica C, 449, 33-40. http://dx.doi.org/10.1016/j.physc.2006.06.048

[15] Krasinkova, M.V. (2008) A New Approach to the Electron State in Transition Metal Oxides. In: Watanabe, T., Ed., Leading-Edge Superconductivity Research Developments, Nova Science Publishers, Inc., 183-193.

[16] Kamihara, Y., Hirano, M., Yanagi, H., Kamiya, T., Saitoh, Y., Ikenaga, E., Kobayashi, K. and Hosono, H. (2008) Physical Review B, 77, 214515. http://dx.doi.org/10.1103/PhysRevB.77.214515

[17] Zhang, C.J., Oyanagi, H., Sun, Z.H., Kamihara, Y. and Hosono, H. (2008) Physical Review B, 78, 214513. http://dx.doi.org/10.1103/PhysRevB.78.214513

[18] Kreyssig, A., Green, M.A., Lee, Y., Samolyuk, G.D., Zajdel, P., Lynn, J.W., Bud’ko, S.L., Torikachvili, M.S., Ni, N., Nandi, S., Leao, J.B., Poulton, S.J., Argyriou, D.N., Harmon, B.N., McQueeney, R.J., Canfield, P.C. and Goldman, A.I. (2008) Physical Review B, 78, 184517. http://dx.doi.org/10.1103/PhysRevB.78.184517

[19] Krasinkova, M.V. (2013) Highly Correlated Electron State and High-Temperature Superconductivity in Iron Pnictides. http://arXiv.org/abs/1302.6002 